TITLE:

\title{
Ligand binding of PDZ domains has various roles in the synaptic clustering of SAP102 and PSD-95.
}

\section{AUTHOR(S):}

Minatohara, Keiichiro; Ichikawa, Sho-Hei; Seki, Tatsuya; Fujiyoshi, Yoshinori; Doi, Tomoko

\section{CITATION:}

Minatohara, Keiichiro ...[et al]. Ligand binding of PDZ domains has various roles in the synaptic clustering of SAP102 and PSD-95.. Neuroscience letters 2013, 533: 44-49

\section{ISSUE DATE:}

2013-01-15

URL:

http://hdl.handle.net/2433/169030

\section{RIGHT:}

(c) 2012 Elsevier Ireland Ltd.; This is not the published version. Please cite only the published version.; この論文は出版社版でありません。引 用の際には出版社版をご確認ご利用ください。 
Ligand binding of PDZ domains has various roles in the synaptic clustering of SAP102 and PSD-95.

Keiichiro Minatohara, Sho-hei Ichikawa, Tatsuya Seki, Yoshinori Fujiyoshi ${ }^{1}$, and Tomoko Doi*

Department of Biophysics, Graduate School of Science, Kyoto University, Kyoto 606-8502 Japan

* corresponding author at: Department of Biophysics, Graduate School of Science, Kyoto University, Kitashirakawa Oiwake-cho, Sakyo-ku, Kyoto 606-8502 Japan.

Tel.: +81 75753 4214; fax: +81 757534117 .

E-mail address: doi@mb.biophys.kyoto-u.ac.jp (T. Doi)

1 Present address: Cellular and Structural Physiology Institute, Nagoya University, Furo-cho, Chikusa, Nagoya 464-8601, Japan.

Abbreviations used: AMPA, $\alpha$-amino-3-hydroxy-5-methylisoxazole-4-propionate; DIV, day in vitro; EGFP, enhanced green fluorescent protein; MAGUK, membrane-associated guanylate kinase; PBS, phosphate-buffered saline; PDZ, PSD-95/discs large/zona occludens-1; PSD, postsynaptic density; PSD-95, postsynaptic density protein-95; SAP102, synapse-associated protein 102; SCI, synaptic clustering index; VGLUT, vesicular glutamate transporter. 


\begin{abstract}
Synapse-associated protein 102 (SAP102) and postsynaptic density-95 (PSD-95) bind to NMDA receptors through PDZ domains and cluster at excitatory postsynaptic sites called postsynaptic densities (PSD). We previously reported that PSD-95 containing mutated PDZ domains incapable of ligand binding clustered at synaptic sites with reduced efficiency. Here, we compared the synaptic clustering of the same series of full-length SAP102 mutants in hippocampal neurons. Unexpectedly, ligand-binding deficient mutant SAP102 showed more efficient synaptic localization than wild-type SAP102. Further, when SAP102-PDZ mutants were co-expressed with either the GluN2A or GluN2B NMDA receptor subunit, both subunits showed decreased synaptic clustering, although the mutants were efficiently targeted to the synapses. This finding suggests that direct binding of NMDA receptors with SAP102 is involved in the efficient targeting of NMDA receptors to the synapses, whereas ligand binding of the PDZ domains is not essential for the synaptic clustering of SAP102.
\end{abstract}

Keywords: SAP102, PSD-MAGUK, PDZ domain, NMDA receptor, postsynaptic density, synaptic clustering. 


\section{Introduction}

Excitatory synapses in the mammalian brain are characterized by a dense network of proteins known as postsynaptic densities (PSD), where the membrane-associated guanylate-kinase (MAGUK) family proteins PSD-95, synapse-associated protein 102 (SAP102), PSD-93, and SAP97 are abundantly expressed and form a protein complex with receptors, ion channels, cytoskeletal proteins, and signaling molecules to regulate signal transduction [11]. PSD-MAGUKs share a common domain structure comprising three PDZ domains in the N-terminus, followed by SH3 and GK domains [3]. They bind directly to the C-termini of the GluN2 subunits of NMDA receptors and the auxiliary subunit of AMPA receptors (TARP) via the PDZ domains. Knock-in mice expressing GluN2B lacking the intracellular C-terminal domain or TARP $\gamma$-8 lacking the C-terminal four residues exhibit perinatal lethality or reduced AMPAR-mediated basal transmission, respectively, suggesting the crucial role of the binding of PSD-MAGUKs with GluN2B or AMPAR in normal signal transduction [12,13]. Thus, PSD-MAGUKs are key molecules for regulating targeting, anchoring, and signaling of receptors and ion channels via protein-protein interactions.

PSD-95 is a dominant PSD-MAGUK protein. The SH3-GK domains and the N-terminal region, including palmitoylated Cys-3 and Cys-5, are essential for efficient targeting and clustering of PSD-95 at synaptic sites [3,16]. Similarly, the N-terminus of SAP102 with no cysteine palmitoylation site is suggested to play a role in synaptic clustering, and the SH3-GK domains of SAP102 are also essential for efficient synaptic targeting through their interaction with mPins, which directly binds the G protein inhibitory subunit and modulates SAP102 trafficking $[3,10]$. SAP102 is highly mobile in dendritic spines, however, compared to PSD-95, suggesting the versatility of MAGUK in their function [17]. The PSD-MAGUKs also have developmentally different roles in the trafficking of NMDA receptors and AMPA receptors to 
synaptic sites, but partially compensate for each other [2]. Thus, how the four PSD-MAGUKs with remarkable similarity play different roles, and the structural bases of their specialization are interesting topics of study.

We previously studied synaptic clustering of PSD-95 containing mutated PDZ domains that are incapable of ligand binding to determine the significance of tandem PDZ domains in synaptic clustering [8]. Our findings revealed that individual PDZ domains independently and additively contribute to the synaptic clustering of PSD-95. In the present study, we introduced the same sets of mutations into SAP102 to assess the effects of ligand binding of PDZ domains on the efficacy of the synaptic clustering of SAP102 in hippocampal neurons. Surprisingly, the clustering of mutated SAP102 was highly efficient, whereas the clustering of GluN2A and GluN2B subunits was impaired by co-expression of the mutated SAP102, suggesting that the mechanisms underlying synaptic clustering are distinct for SAP102 and PSD-95, and that SAP102 has a fundamental role in trafficking the NMDA receptor complex to the synaptic sites. 


\section{Materials and Methods}

\subsection{DNA constructs}

The mutated PSD-95 cDNAs fused with enhanced green fluorescent protein (EGFP) were described previously [8]. The cDNA of SAP102 (GI:118131177) coding 849 amino acids was prepared from a mouse brain full-length cDNA library (Clontech) and introduced into the chicken $\beta$-actin promoter-based expression vectors, pCA-flag and pCA-EGFP, kindly provided by Drs. H. Niwa and M. Takeichi (RIKEN, Center for Developmental Biology). The ligand-binding deficient PDZ1 domain (1d) of SAP102 was prepared by introducing S162N and A164 V mutations and deleting I167 to V172. Similarly, the mutant PDZ2 domain (2d) contained S257N and A259V mutations and a deletion of I262 to I267. The mutated PDZ3 domain (NS) of SAP102 contained an N417S mutation. The myc-tag (EQKLISEEDL) was inserted between N35 and I36 of the mouse GluN2A subunit and between A53 and H54 of the GluN2B subunit. These cDNAs were introduced into the pCA expression plasmid.

\subsection{Immunoprecipitation}

The full-length mutant SAP102 was co-expressed with myc-tagged GluN2B in COS cells and cell lysates, prepared in 1\% Triton X100, 50 mM Tris-HCl (pH7.5), 0.1 M NaCl, 1 mM EDTA, and protease inhibitors, were incubated with $5 \mu \mathrm{g}$ myc antibody overnight at $4^{\circ} \mathrm{C}$. The mixtures were further incubated with $30 \mu$ l Protein $\mathrm{G}$ agarose for $1 \mathrm{~h}$ at $4^{\circ} \mathrm{C}$. The extracts from the Protein G agarose with sodium dodecyl sulfate sample buffer were analyzed by $7.5 \%$ sodium dodecyl sulfate-polyacrylamide gel electrophoresis and subsequent immunoblotting with the anti-myc antibody 9E10 (Roche Applied Science), and the anti-GFP antibody JL-8 (Clontech, Takara Bio Inc.). 


\subsection{Hippocampal neural cell cultures and transfection}

Rat hippocampal dissociated neurons were prepared from embryonic day 18 Wister rats, as described previously [7] and transfected with cDNAs using a Rat Neuron Nucleofector kit (Amaxa Biosystems, Gaithersburg, MD) according to the manufacturer's instructions. Transfected neurons were then plated directly onto poly-L-lysine (Sigma, St. Louis, MO)-coated culture dishes at a density of $3 \times 10^{3}$ cells $/ \mathrm{cm}^{2}$ and incubated in Neurobasal medium (Invitrogen, Grand Island, NY) supplemented with B27 (Invitrogen) and $1 \mathrm{mM} \mathrm{L-glutamine.} \mathrm{At} \mathrm{15} \mathrm{to} 17$ days in vitro (DIV), cells were fixed in phosphate-buffered saline (PBS) containing 4\% paraformaldehyde/4\% sucrose. The fixed cells were quenched in $1 \mathrm{mM}$ glycine/PBS, permeabilized with $0.2 \%$ Triton $\mathrm{X}-100 / \mathrm{PBS}$ for $10 \mathrm{~min}$ at room temperature, thoroughly washed with PBS, incubated in blocking solution (3\% bovine serum albumin/PBS) for $30 \mathrm{~min}$ at room temperature, and then incubated overnight at $4^{\circ} \mathrm{C}$ with primary antibodies diluted in the blocking buffer as described below, washed three times with PBS, and incubated with AlexaFluor-conjugated secondary antibodies (Molecular Probes) at a 1:500 dilution in blocking buffer. Antibodies were purchased and diluted as follows: mouse monoclonal anti-synaptophysin (Sigma, 1:1000), rabbit polyclonal anti-VGLUT1 (Synaptic Systems, Goettingen Germany; 1:1000), and AlexaFluor-conjugated anti-rabbit and anti-mouse antibodies (Invitrogen; 1:500). All animal experiments were performed according to the guidelines approved by the Animal Experimentation Committee of Kyoto University.

\subsection{Quantitative microscopy and image analysis}

All immunofluorescence images were acquired using a cooled charge-coupled device camera, the Cool SNAP HQ (Photometrics) controlled by Meta Morph software (Molecular Devices), 
mounted on a BX50 upright microscope (Olympus) with 100x (N.A.1.35) or 40x (N.A.0.85) objectives. For quantification, because the intensity values derived from fused GFP varied due to overexpression, neurons that expressed each mutant-GFP within the following average intensity values in the dendritic shafts were randomly picked; 102-WT, 102-1d2d, and 102-1d2dNS: 50-220; 95-WT, 95-1d2d, 95-1d2dNS: 100-300 in a 12-bit dynamic range, 0-4096, at a fixed gain setting. Most of the cells with a normal morphology fell within these values.

Calculation of the synaptic clustering index (SCI) shown in Fig. 2 was performed as described previously [8]. Briefly, synaptic PSD-95 or SAP102 clusters were defined as 0.3 to $1.0 \mu \mathrm{m}$ diameter spots of increased GFP fluorescence, at least twice as bright as the dendritic shaft, which were juxtaposed to synaptophysin-immunopositive puncta. To calculate the SCI, after subtracting the background, the maximal intensity of each synaptic cluster was divided by the mean intensity of the proximal parent dendritic shaft. In each neuron, 20 to 30 synaptic clusters that colocalized with synaptophysin were selected to calculate the SCI. A total of 6 to 10 cells was analyzed for each transfected construct. Three independent trials of transfection with a series of mutants were performed, and the same tendency shown in Fig. 2 was observed for each imaging and quantification. All data are expressed as the mean \pm SEM. For statistical analysis, the SCI measurement was evaluated by one-way ANOVA with Dunnett's multiple comparison post hoc test.

For quantification of images of neurons co-expressing SAP102 with GluN2A or GluN2B in Fig. 3, we calculated the mean SCI (mSCI) using the following averaging method with Meta Morph software, because calculation of the SCIs for both of SAP102 and GluN2A or GluN2B for individual synapses of each mutant construct was time- and labor-intensive. Basically, the mSCI was calculated as the mean synaptic cluster intensity at the sites juxtaposed to the presynaptic markers divided by the mean dendritic shaft intensity. The synaptic clusters were 
defined as the areas between 10 and 300 pixels with an intensity greater than $10 \%$ of the maximum in a neuron that were colocalized with a presynaptic marker, VGLUT1. Mean synaptic intensity was calculated by dividing the total synaptic intensity by the corresponding total synaptic area. Mean dendritic shaft intensity was calculated by dividing the total dendritic shaft intensity by the total dendritic shaft area. The mSCI was defined as the ratio of the mean spine intensity to the mean dendritic shaft intensity. A total of 6 to 10 cells was analyzed for each transfected construct. Three independent trials were performed and the same tendency shown in Fig. 3 was observed for each imaging and quantification. The mSCIs for a series of SAP102 mutants (Fig. 3) provided results comparable to those obtained above in the SCIs (Fig. 2), although the absolute values differed. 


\section{Results}

\subsection{Construction of the full-length SAP102 mutants containing PDZ domains with impaired ligand binding.}

In the mutant PDZ domains, PDZ1d and PDZ2d of SAP102, two exchanged residues in the $\beta B$ sheet and six deleted residues in the $\beta \mathrm{B} / \beta \mathrm{C}$ loop near the ligand binding site were designed to mimic the PDZ3 structure to retain the overall structure with decreased ligand binding activity (Fig.1a, b) [1,14]. The same kinds of mutant PDZ domains of PSD-95 are unable to bind the original ligand, GluN2B, and CRIPT, known as a PDZ3 ligand [4,8]. The N417S mutation in the PDZ3 of SAP102 corresponds to the N326S mutation in the PDZ3 of PSD-95, which also results in significantly reduced ligand binding activity [8]. These mutated PDZ domains, PDZ1d, PDZ2d, and PDZ3-NS, were systematically introduced into the full-length SAP102 fused with GFP at the C-terminus. The series of SAP102-GFP mutants used in this study is shown in Figure 1c. The reduced ligand binding activity of the full-length SAP102 PDZ-mutants was verified by immunoprecipitation with GluN2B co-expressed in COS cells (Fig. 1d).

\subsection{Lack of ligand binding of PDZ domains resulted in highly efficient synaptic clustering of SAP102.}

To examine the role of ligand binding of PDZ domains in synaptic clustering, the ligand-binding deficient SAP102 PDZ-mutants (102-1d2d and 102-1d2dNS) were transfected along with the wild-type (102-WT) in dissociated hippocampal neurons, which were further cultured for 15 to 17 days. Neurons were fixed and immunostained with a presynaptic marker, synaptophysin. The full-length wild-type SAP102 and PDZ-mutants fused with GFP formed distinguishable clusters juxtaposed to immunoreactive sites of synaptophysin, as observed for PSD-95 [8]. The 
previously described SCI was used to quantitatively measure the clustering efficiency at the postsynaptic site [8]. Basically, the SCI was calculated as the peak GFP pixel intensity at the clusters juxtaposed to the presynaptic marker, divided by the mean GFP pixel intensity of the adjacent dendritic shaft. Neurons that expressed each mutant-GFP within the certain range of intensity in the dendritic shafts were chosen to compare (see Materials and Methods). Most of the clusters that colocalized with synaptophysin were assumed to be "synaptic", although a subset of trafficking organelles potentially localized with a presynaptic marker by chance.

Unexpectedly, 102-1d2d and 102-1d2dNS with mutated PDZ domains clustered at synapses extremely efficiently compared to 102-WT (Fig.2A, b, c), whereas the same sets of PSD-95 mutants, 95-1d2d and 95-1d2dNS, substantially remained in the dendritic shaft and clustered at synapses inefficiently compared to the wild-type 95-WT (Fig. 2B, b, c). The average SCI values of $102-1 \mathrm{~d} 2 \mathrm{~d}$ and $102-1 \mathrm{~d} 2 \mathrm{dNS}$ were $15.22 \pm 1.49$ and $16.77 \pm 1.82$, respectively, and the wild-type SCI was 8.19 \pm 0.79 (Fig. 2A, j). The SAP102 PDZ-mutants had approximately 1.5- to 2-fold more efficient synaptic clustering than the wild-type, whereas synaptic clustering of PSD-95 PDZ-mutants was decreased to approximately one-half (Fig. 2B, j). Thus, ligand binding of PDZ domains is important for efficient targeting to the synapses and/or stability in the synapses for PSD-95, but not SAP102, suggesting that these molecules use different pathways for trafficking and/or anchoring to synapses. Moreover, ligand binding of PDZ domains is not essential for the synaptic clustering of SAP102.

\subsection{Ligand-binding mutants of SAP102 decreased the synaptic clustering of GluN2A and GluN2B.}

To explore the roles of PDZ binding of SAP102, we further co-expressed SAP102-PDZ mutants with the myc-tagged NMDA receptor subunits GluN2A or GluN2B in cultured hippocampal 
neurons. At DIV 15, neurons were fixed and immunostained with anti-myc antibody and anti-VGLUT1 antibody, as a presynaptic marker. VGLUTI, which could be stained specifically by a rabbit antibody, was used as an excitatory presynaptic marker for double immunostaining, because the anti-myc antibody was a mouse antibody.

As noted above, the SAP102-PDZ mutants efficiently localized to synapses with a low intensity in dendritic shafts. GluN2A or GluN2B subunit localization at synapses probed with the anti-myc antibody, however, was substantially decreased (Fig. 3). For these images, we used an averaging method to analyze the mSCI described in the Materials and Methods that could be performed within a relatively shorter time. Basically, mSCI was calculated as the mean synaptic cluster intensity at the sites juxtaposed to presynaptic markers divided by the mean dendritic shaft intensity. While the mSCIs of the SAP102 PDZ-mutants 102-1d2d and 102-1d2dNS $\left(\mathrm{mSCI}_{1 \mathrm{~d} 2 \mathrm{~d}}, 6.75 \pm 0.56 ; \mathrm{mSCI}_{1 \mathrm{~d} 2 \mathrm{dNS}}, 7.96 \pm 0.33\right)$ were approximately 1.5 -fold as efficient as that of the wild-type (mSCI $\mathrm{WT}_{\mathrm{W}}, 5.08 \pm 0.39$ ), the mSCIs of GluN2B were decreased to two-thirds by

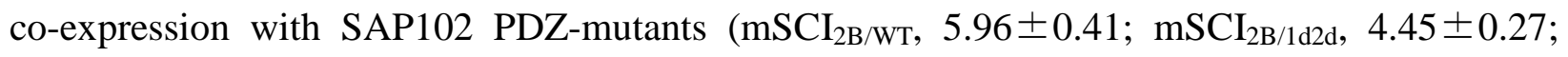
$\mathrm{mSCI}_{2 \mathrm{~B} / 1 \mathrm{~d} 2 \mathrm{dNS}}, 4.38 \pm 0.25$, Fig. 3B). Co-expression of SAP102 with GluN2A produced similar results. While the mSCIs of SAP102 PDZ-mutants $\left(\mathrm{mSCI}_{1 \mathrm{~d} 2 \mathrm{~d}}, 6.11 \pm 0.66 ; \mathrm{mSCI}_{1 \mathrm{~d} 2 \mathrm{dNS}}, 6.21 \pm\right.$ 0.35) were higher than that of the wild-type (mSCI $\left.\mathrm{WT}_{\mathrm{W}}, 4.46 \pm 0.35\right)$, the mSCIs of GluN2A were decreased by co-expression with SAP102 PDZ-mutants (mSCI 2A/wT, $4.73 \pm 0.31$ mSCI $_{2 \mathrm{~A} / 1 \mathrm{~d} 2 \mathrm{~d}}$,

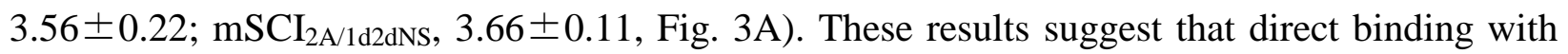
the PDZ domains of SAP102 enables efficient synaptic clustering of the GluN2 subunits, but suppresses synaptic clustering of SAP102 .

\section{Discussion}

PSD-MAGUK family scaffolding proteins have highly similar domain architectures. Here, we 
explored the different roles of PDZ ligand-binding in the synaptic clustering of SAP102 and PSD-95. The lack of ligand-binding induced by specific mutations of the PDZ domains led to significantly enhanced synaptic clustering of full-length SAP102, contrary to that of mutant PSD-95. This distinct characteristic might reflect their different trafficking and/or anchoring mechanisms, their diverse roles as scaffolding proteins, and therefore their involvement in synaptic transmission.

To design PDZ mutations that impair ligand-binding, a good candidate is the conserved GLGF motif in the $\beta A-\beta B$ loop, which plays a key role in carboxylate-ligand binding [1]. To retain the overall domain structure, however, our strategy was to mimic the PDZ3 structure in the PDZ2 domain, because PDZ3 binds PDZ2 ligands with lower affinity.

The efficient synaptic clustering of SAP102-PDZ mutants indicates that the N-terminal region and SH3-GK domains are sufficient for synaptic localization of SAP102. The mechanisms involving mPins may drive SAP102-PDZ mutants to the synapses. Why was SAP102 transported to synapses more efficiently when the PDZ ligand-binding abilities were lost? In synaptic delivery, NMDA receptors are transported along microtubules in vesicle-associated protein complexes containing SAP102 and early endosomal antigen 1 . This complex cycles in and out of the dendritic plasma membrane during transport from the cell body to distal dendritic spines [15]. Therefore, due to the lack of PDZ binding, SAP102 released from the transport complex of NMDA receptors could freely move to the synapses in association with mPins.

We found that impaired direct interactions of GluN2A or GluN2B with SAP102 resulted in inefficient synaptic clustering of NMDA receptor subunits. The subunit assembly, intracellular trafficking, synaptic delivery, and activity-dependent exo- and endocytosis in the synapses of NMDA receptors are regulated by a number of mechanisms [5,6]. It is not clear from our results 
which processes were impaired by the lack of direct binding. SAP102 also directly binds to Sec8, a component of the exocyst complex, through a PDZ domain. Colocalization of SAP102 and Sec8 is observed in the rough endoplasmic reticulum in the soma [9]. Interaction of SAP102 with GluN2B and mPins occurs early in the secretory pathway [10]. Therefore, the direct binding of SAP102 with GluN2 subunits supports the efficient delivery of the NMDA receptor from the soma to the synaptic sites. In addition, SAP102 is the only PSD-MAGUK family protein that does not self-associate, while PSD-93, PSD-95 and SAP97 oligomerize through the N-terminal region. The monomeric structure of SAP102 might be suitable for transporting NMDA receptor complexes to synaptic sites.

The findings of the present study revealed that ligand binding of PDZ domains has distinct roles in the synaptic clustering of SAP102 and PSD-95. These findings raise the possibility that PSD-MAGUKs have different roles in neuronal activities starting from synaptogenesis, and that the efficacy of synaptic clustering of SAP102 is modulated by the levels of PDZ-ligands in synaptic trafficking. Further studies are required to elucidate their diverse functions, which will help to further dissect and elucidate the molecular mechanisms of synapse formation as well as synaptic transmission.

\section{Acknowledgements}

We would like to thank Ms. M. Tanaka and Dr. K. Tani for figure preparation and Dr. M. Goda for suggestions on the image analysis. This work was supported by a JSPS Grant-in-Aid for scientific research 19300128 and 22227004, and the New Energy and Industrial Technology Development Organization of Japan. 


\section{References}

[1] D.A. Doyle, A. Lee, J. Lewis, E. Kim, M. Sheng, R. MacKinnon, Crystal structures of a complexed and peptide-free membrane protein-binding domain: molecular basis of peptide recognition by PDZ, Cell 85 (1996) 1067-1076.

[2] G.M. Elias, L.A.B. Elias, P.F. Apostolides, A.R. Kriegsteinand, R.A. Nicoll Differential trafficking of AMPA and NMDA receptors by SAP102 and PSD-95 underlies synapse development, Proc. Natl. Acad. Sci. USA 105 (2008) 20953-20958.

[3] L. Funke, S. Dakoji, D.S. Bredt, Membrane-associated guanylate kinases regulate adhesion and plasticity at cell junction, Annu. Rev. Biochem. 74 (2005) 219-245.

[4] F. Imamura, S. Maeda, T. Doi, Y. Fujiyoshi, Ligand binding of the second PDZ domain regulates clustering of PSD-95 with the Kv1.4 potassium channel. J. Biol. Chem. 277 (2002) 3640-3646.

[5] O. Jeyifous, C.L. Waites, C.G. Specht, S. Fujisawa, M. Schubert, E.I. Lin, J. Marshall, C. Aoki, T. Silva, J.M. Montgomery, C.C. Garner, Green W.N., SAP97 and CASK mediate sorting NMDA receptors through a previously unknown secretory pathway, Nat. Neurosci. 12 (2009) 1011-1019.

[6] C.G. Lau, R.S. Zukin, NMDA receptor trafficking in synaptic plasticity and neuropsychiatric disorders, Nat. Rev. Neurosci. 8 (2007) 413-426.

[7] Y. Murata, T. Doi, H. Taniguchi, Y. Fujiyoshi, Proteomic analysis revealed a novel synaptic proline-rich membrane protein (PRR7) associated with PSD-95 and NMDA receptor, Biochem. Biophys. Res. Commun. 327 (2005) 183-191.

[8] M. Nonaka, T. Doi, Y. Fujiyoshi, S. Takemoto-Kimura, H. Bito, Essential contribution of the ligand-binding $\beta \mathrm{B} / \beta \mathrm{C}$ loop of PDZ1 and PDZ2 in the regulation of postsynaptic clustering, scaffolding, and localization of postsynaptic density-95, J. Neurosci. 26 (2006) 763-774. 
[9] N. Sans, K. Prybylowski, R.S. Petralia, K. Chang, Y.-X. Wang, C. Racca, S. Vicini, R.J. Wenthold, NMDA receptor trafficking through an interaction between PDZ proteins and the exocyst complex, Nat. Cell Biol. 5 (2003) 520-530.

[10] N. Sans, P.Y. Wang, Q. Du, R.S. Petralia, Y.-X. Wang, S. Nakka, J.B. Blumer, I.G. Macara, R.J. Wenthold, mPins modulated PSD-95 and SAP102 trafficking and influences NMDA receptor surface expression, Nat. Cell Biol. 7 (2005) 1179-1190.

[11] M. Sheng, C.C. Hoogenraad, The postsynaptic architecture of excitatory synapses: a more quantitative view, Annu. Rev. Biochem. 76 (2007) 823-847.

[12] R. Sprengel, B. Suchanek, C. Amico, R. Brusa, N. Burnashev, A. Rodov, Ø. Hvalby, V. Jensen, O. Paulsen, P. Andersen, J.J. Kim, R.F. Thompson, W. Sun, L.C. Webster, S.G.N. Grant, J. Eilers, A. Konnerth, J. Li, J.O. McNamara, P.H. Seeburg, Importance of the intracellular domain of NR2 subunits for NMDA receptor function in vivo, Cell 92 (1998) 279-289.

[13] A. Sumioka, T.E. Brown, A.S. Kato, D.S. Bredt, J.A. Kauer, S. Tomita, PDZ binding of TARP $\gamma-8$ controls synaptic transmission but not synaptic plasticity, Nat. Neurosci. 14 (2011) $1410-1412$.

[14] H. Tochio, F. Hung, M. Li, D.S. Bredt, M. Zhang, Solution structure and backbone dynamics of the second PDZ domain of postsynaptic density-95, J. Mol. Biol. 295 (2000) 225-237.

[15] P. Washbourne, X.-B. Liu, E.G. Jones, A.K. McAllister, Cycling of NMDA receptors during trafficking in neurons before synapse formation, J. Neurosci. 24 (2004) 8253-8264. [16] W. Xu, O.M. Schlüter, P. Steiner, B.L. Czervionke, B. Sabatini, R.C. Malenka, Molecular dissociation of the role of PSD-95 in regulating synaptic strength and LTD, Neuron 57 (2008) 248-262.

[17] C.-Y. Zheng, R.S. Petralia, Y.-X. Wang, B. Kachar, R.J. Wenthold, SAP102 is a highly 
mobile MAGUK in spines, J. Neurosci. 30 (2010) 4757-4766. 


\section{Figure legends.}

Fig. 1 (a) Ribbon diagrams showing the three-dimensional folds of the PDZ2 (left) and PDZ3 (right) domains of PSD-95. The elements of the secondary structures are labeled. The diagrams are based on the data from the worldwide Protein Data Bank (PDZ2 PDB ID: 1QLC, PDZ3 PDB ID: 1BE9). The basic backbone structures of PDZ2 and PDZ3 are very similar, except the loop length between $\beta B$ and $\beta C$. To model the PDZ3 structure, the region shown in magenta in the $\beta B-\beta C$ loop of PDZ2 was deleted and the two residues shown in red in the $\beta B$ were mutated. (b) Amino acid sequences around the $\beta \mathrm{B}-\beta \mathrm{C}$ region of the PDZ1, PDZ2 and PDZ3 domains of SAP102. The six deleted residues in the $\beta B-\beta C$ loop are boxed. The mutated residues in $\beta B$ are boxed and marked with a bold font. (c) The wild-type and mutant full-length constructs of SAP102 and PSD-95 used in this study. '1d' and '2d' indicate the mutated PDZ1 and PDZ2 domains, respectively. 'NS' in the mutated PDZ3 domain indicates a single mutation. All the constructs were fused with GFP at the C-terminus. (d) The mutated PDZ domains abolished the interaction of SAP102 with GluN2B. The myc-GluN2B co-expressed with the wild-type or mutant SAP102 in COS cells was immunoprecipitated with a myc antibody. The immunoprecipitated GluN2B and SAP102 bound to GluN2B were analyzed by immunoblotting. Lane 1, input; lane 2, passed fraction of protein $G$ resin; lane 3, eluate from protein $G$ resin. Mutant SAP102 containing 1d and 2d domains bound to GluN2B was hardly detected in this system.

Fig. 2 Ligand-binding deficient PDZ mutations enhance the synaptic clustering of SAP102. GFP fluorescent and synaptophysin immunofluorescent images were obtained from transfected hippocampal neurons expressing the wild-type and mutant full-length SAP102-GFP (A) and PSD-95-GFP (B). Ratios of fluorescence intensity in the synaptic clusters vs. that in the parent 
dendritic shaft were compared. Panels (a), (b) and (c) are GFP fluorescence images of wild-type, 1d2d and 1d2dNS MAGUKs, respectively. Panels (d), (e), (f) are magnified images of (a), (b), (c), respectively. Panels (g), (h), (i) are overlaid frames of GFP (green) and synaptophysin (red; indicating presynaptic terminals) images. Panels $(\mathrm{a} \sim \mathrm{c})$ and panels $(\mathrm{d} \sim \mathrm{i})$ are images with the same magnification, respectively. Scale bars indicate $5 \mu \mathrm{m}$. Histograms (j) in (A) and (B) show the synaptic clustering index of SAP102 and PSD-95, respectively. Each dataset was statistically analyzed using a one-way ANOVA, with a post hoc Dunnett's test. **: $p<0.01$ and *: $p<0.05$ vs. WT.

Fig. 3. Ligand-binding deficient mutations of SAP102 lead to reduced synaptic clustering of GluN2 subunits. The ligand-binding mutants of SAP102 were co-expressed with myc-tagged GluN2A (A) or with GluN2B (B) in hippocampal neurons. Cultured neurons were fixed at DIV15 and labeled with anti-myc and anti-VGLUT1 antibodies. Panels (a), (b), and (c) are GFP fluorescence images of 102WT, 102-1d2d, and 102-1d2dNS, respectively. Panels (d), (e), and (f) show respective myc-labeled images. Panels (g), (h), and (i) are superimposed images of GFP (green), myc-labeled (red), and VGULT1-labeled (blue), respectively. The mSCIs for ligand-binding deficient mutants (j) were similar to the results shown in Fig. 2. The mSCIs for GluN2A (k) in A and GluN2B (k) in B were reduced when co-expressed with ligand-binding deficient SAP102 mutants. ${ }^{* *}: \mathrm{p}<0.01$ and ${ }^{*}: \mathrm{p}<0.05$ vs. the respective WT, as described in the legend for Fig. 2. Scale bars: $5 \mu \mathrm{m}$. 
(a)

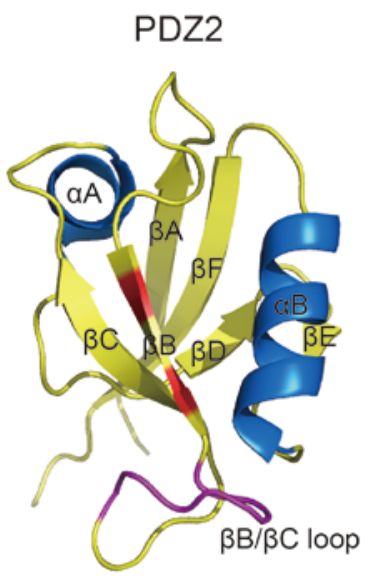

(b)

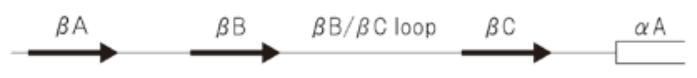

PDZ1 (149) EI ILERGNSGLGFS IAGG IDNPHVPDDPG IF I TK I IPGGAAA . PDZ2 (244) EVNLLKGPKGLGFS I AGG I GNQHI PGDNS IY I TK I IEGGAAQ * PDZ3 (404) EI ILHKGSTGLGFNIVGG----EDGEG IFVSF ILAGGPAD $\cdots$

Fig.1 (c)

SAP102-GFP
$102 \mathrm{WT}$
$102-1 \mathrm{~d} 2 \mathrm{~d}$
$102-1 \mathrm{~d} 2 \mathrm{dNS}$

PSD-95-GFP

95WT

$95-1 \mathrm{~d} 2 \mathrm{~d}$

95-1d2dNS
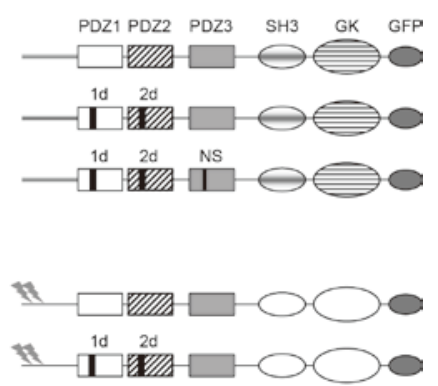

11 1d $2 \mathrm{~d}$ NS

(d)

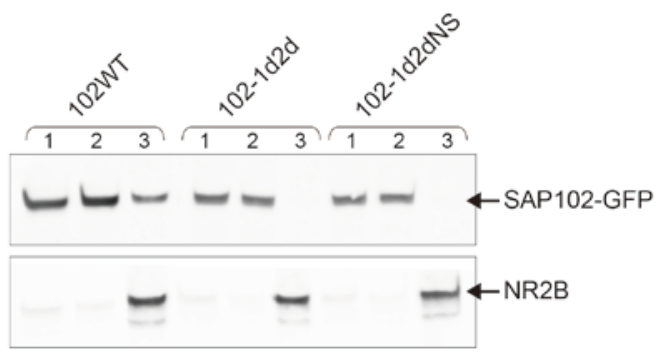




\section{A. SAP102}
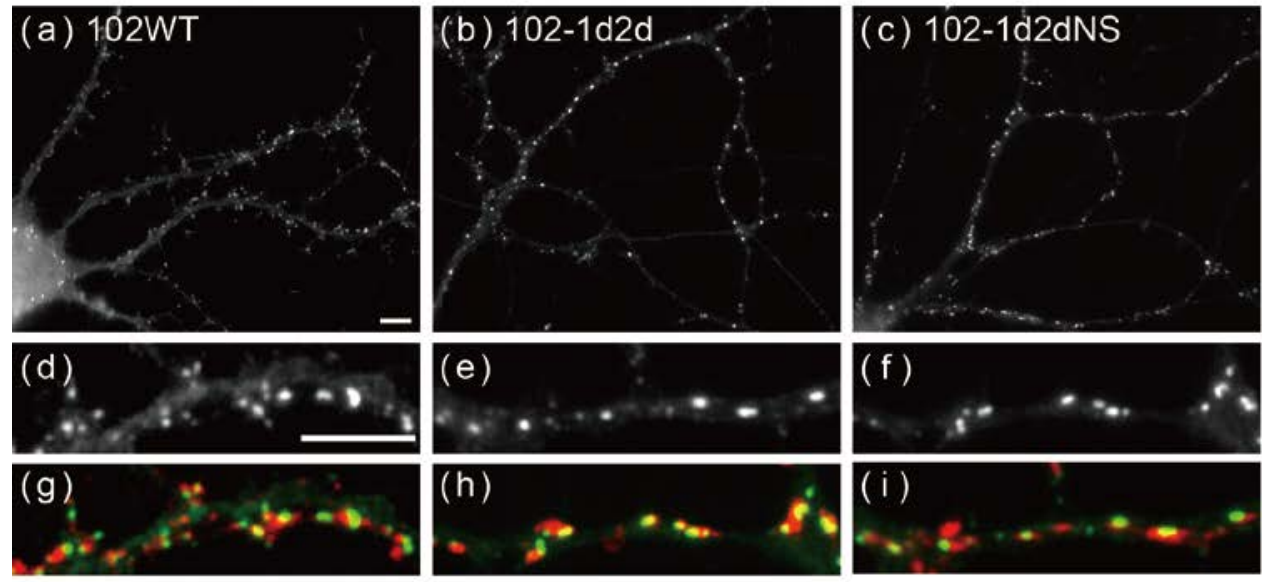

(j) SAP102

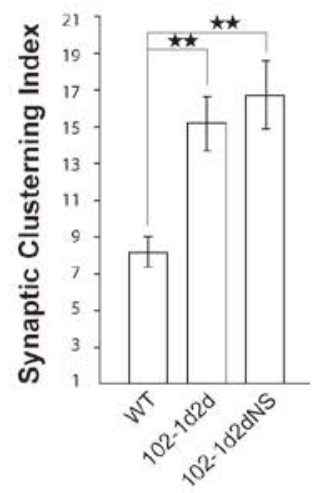

B. PSD-95
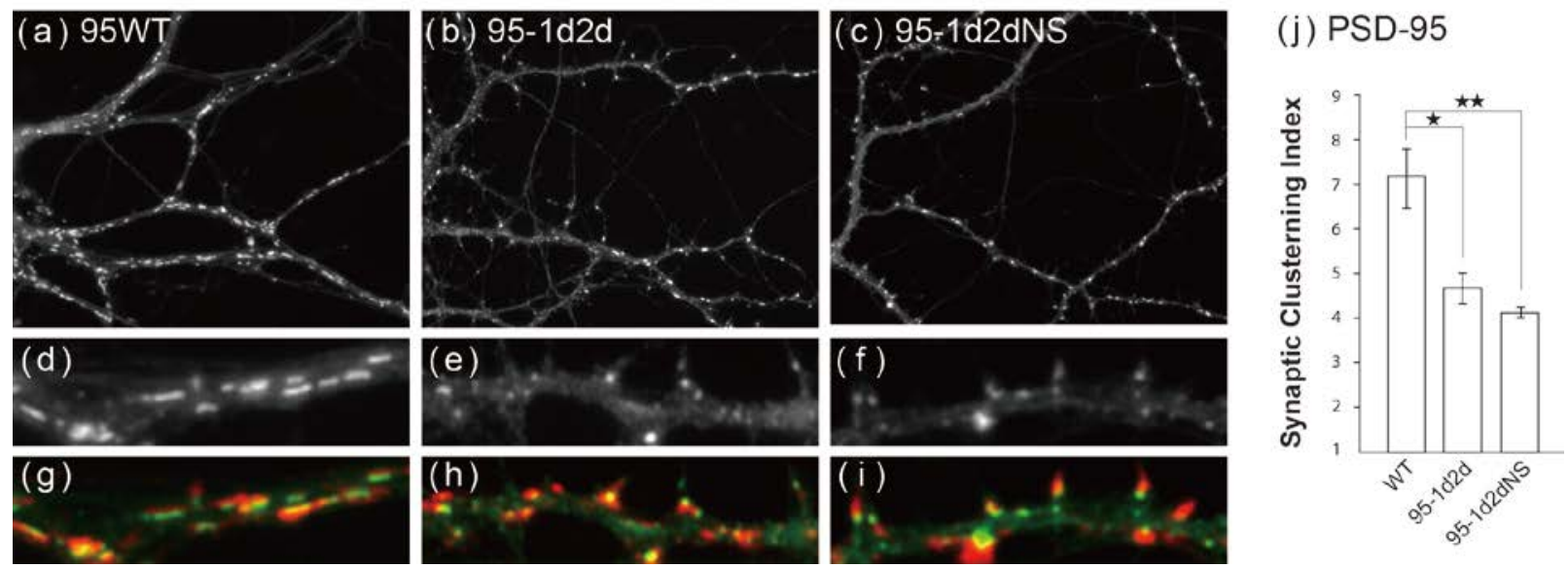

Fig.2 


\section{A. SAP102-GluN2A}

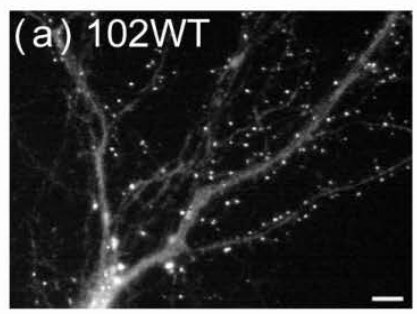

(d) GluN2A
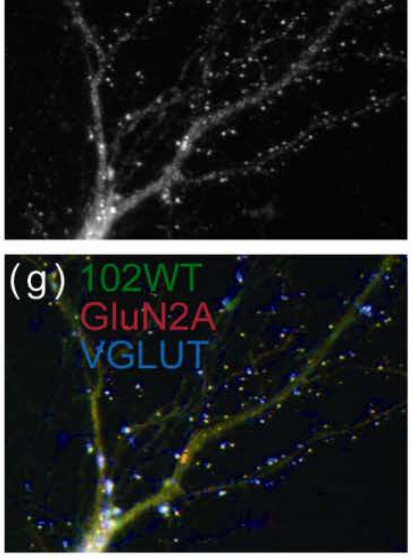

B. SAP102-GluN2B
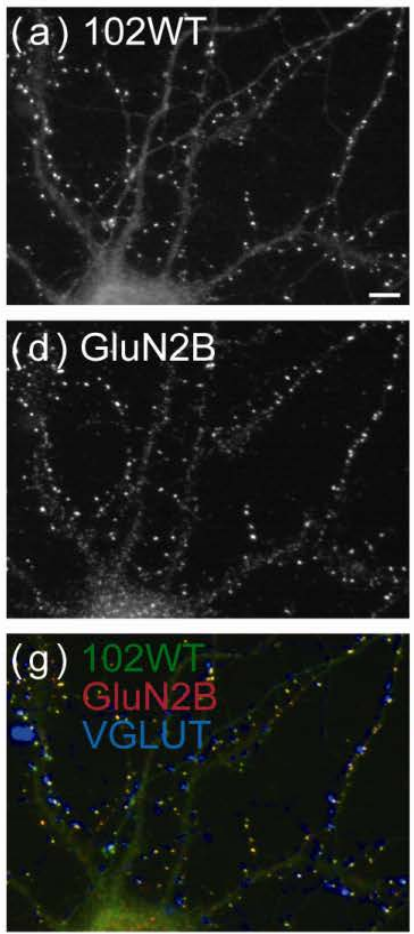
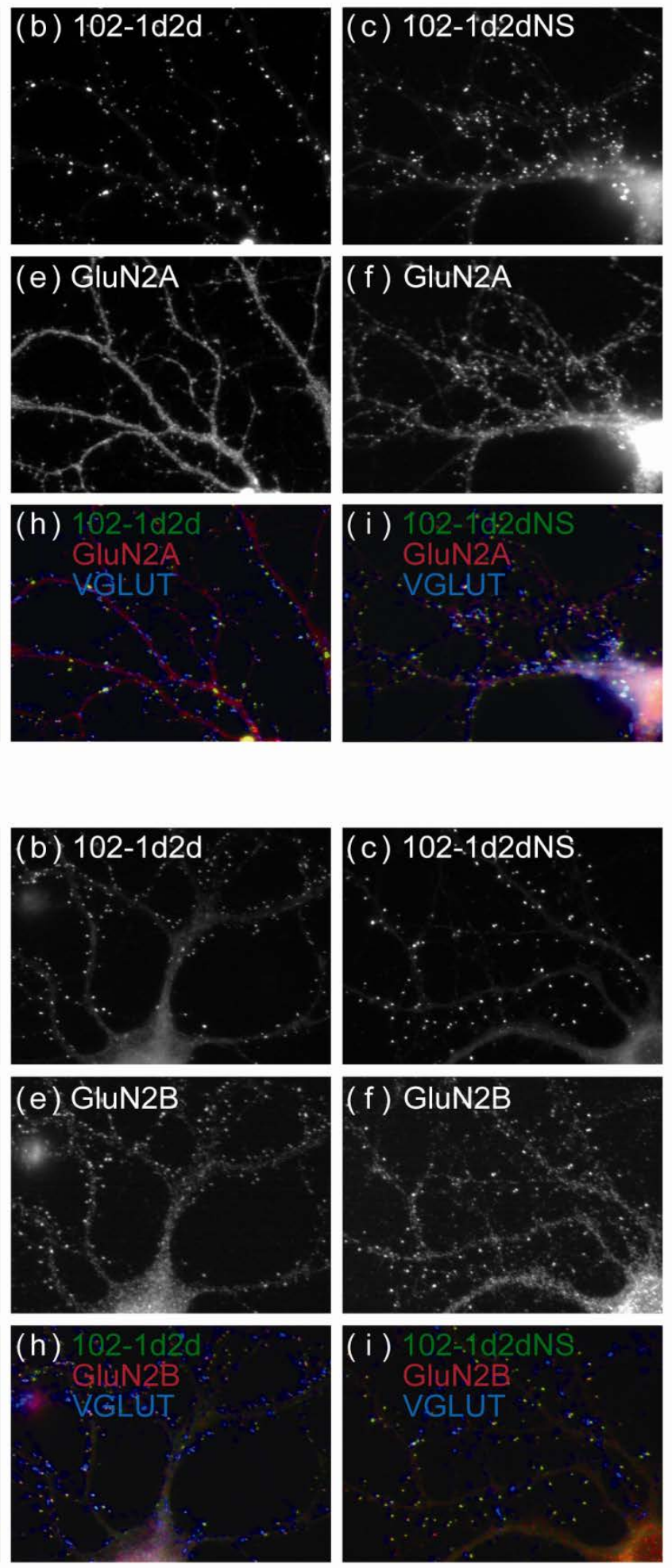
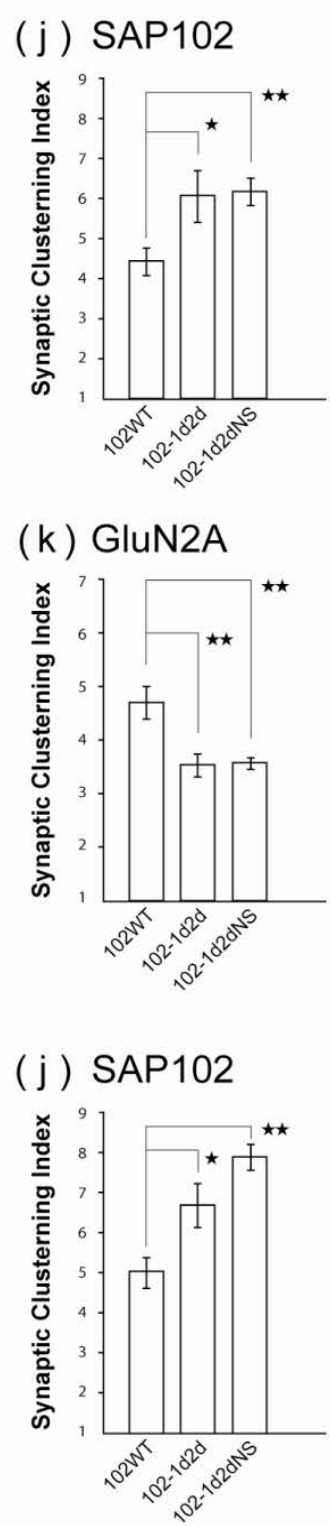

(k) GluN2B

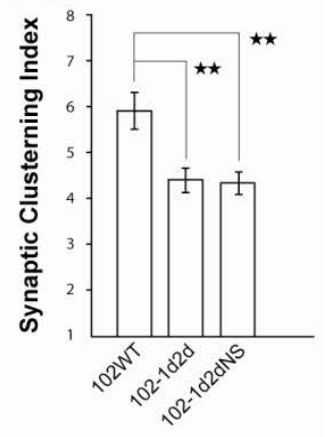

Fig.3 